\title{
The Results of Thrombolytic Treatment in Patients With High-risk Pulmonary Embolism
}

\author{
Ali Ekber Atas ${ }^{1}$, Atilla Sarac ${ }^{2}$, Adem Dirican ${ }^{3}$, Nazlı Topbası $^{4}$, Tugce Uzar ${ }^{5}$, Ladan Rastgar ${ }^{6}$, \\ Pankina Ekaterina ${ }^{7}$, Sevket Ozkaya ${ }^{4}$ \\ ${ }^{1}$ Department of Cardiology, Samsun Medicalpark Hospital, Samsun, Turkey \\ ${ }^{2}$ Department of Cardiovascular Surgery, Samsun Medicalpark Hospital, Samsun, Turkey \\ ${ }^{3}$ Department of Pulmonary Medicine, Samsun Medicalpark Hospital, Samsun, Turkey \\ 4 Department of Pulmonary Medicine, Faculty of Medicine, Bahcesehir University, Istanbul, Turkey \\ ${ }^{5}$ Medical Student/Intern, Faculty of Medicine, Bahcesehir University, Istanbul, Turkey \\ ${ }^{6}$ Faculty of Medicine, Bahcesehir University, Istanbul, Turkey \\ ${ }^{7}$ Department of Pulmonary Medicine, Faculty of Medicine, Bashkir State Medical University, Ufa, Russia
}

\begin{abstract}
Background: Mortality rates due to massive pulmonary embolism (PE) are much higher than estimated. Although thrombolytic therapy is controversial, it can be a life-saving procedure and can be safely used in patients with massive PE. Study aim: We aimed to share the results of thrombolytic treatment in patients with massive PE. Material and methods: We retrospectively evaluated 72 patients with PE admitted between January 2010 and April 2018 to the Department of Pulmonary Medicine, VM Medicalpark Samsun Hospital, Samsun, Turkey. The data of patients who received thrombolytic treatment were retrospectively analyzed. Results: The female to male ratio was $24 / 48$, with a mean age of $62.7 \pm 12.6$ (minimum 27 , maximum 88 ) years. The diagnosis of massive PE was established with echocardiography in all patients and was confirmed via pulmonary CT angiography in 45 patients $(62.5 \%)$ who presented an appropriate clinical status for this imaging technique. The most common symptoms were dyspnea (90.3\%), chest pain (83.3\%), and syncope (40.2\%). The S1Q3T3 electrocardiography pattern was noted in $82 \%$ of patients, who rapidly recovered after thrombolytic therapy. Cardiopulmonary arrest was seen in 25 patients $(37.2 \%)$, and thrombolytic treatment was administered during cardiopulmonary resuscitation in $18 \%(n=13)$ of patients. The survival rate was $30.7 \%(n=4)$ in patients with cardiopulmonary arrest who received thrombolytic treatment in the emergency room. The complications of rt-PA treatment included minor hemorrhages in $6.4 \%(n=5)$, major hemorrhages in $2.7 \%(n=2)$, and allergic reactions in $1.3 \%(n=1)$ of patients. None of the patients had deceased as a complication of thrombolytic treatment. The overall mortality rate was $26.2 \%(n=19)$, and $12.5 \%(n=9)$ of the patients have died in first 24 hours after thrombolysis. Conclusions: We concluded that the risk factors, ECG, and echocardiography are key indicators for the suspicion of massive PE in patients with hemodynamic shock. Based on our experience, early thrombolytic therapy is a life-saving intervention in patients with diagnosed and/or suspected massive PE.
\end{abstract}

Keywords: massive pulmonary embolism, thrombolytic therapy, alteplase, radiology

\section{ARTICLE HISTORY}

Received: June 29, 2019

Accepted: August 19, 2019

\section{CORRESPONDENCE}

\section{Sevket Ozkaya}

Department of Pulmonary Medicine, Faculty of Medicine, Bahcesehir University Yıldız, Osman Paşa Mektebi Sk. No. 4A 34353 Beşiktaş/Istanbul, Turkey Tel: +90 5324741309 E-mail: ozkayasevket@yahoo.com 


\section{INTRODUCTION}

Pulmonary embolism (PE) is increasingly recognized as a cause of significant morbidity and mortality. Patients with $\mathrm{PE}$ are commonly classified into massive (high-risk), submassive (intermediate-risk), and low-risk PE to help determine the required treatment. Risk stratification scores are used to determine the risk of complications and associated mortality. Massive or high-risk PE is defined as suspected or confirmed PE in the presence of shock, sustained hypotension, absence of peripheral pulse, or persistent profound bradycardia. Submassive PE is defined as suspected or confirmed PE with right ventricular dysfunction in the absence of shock. ${ }^{1,2}$ According to various studies, the rates of mortality due to massive PE are much higher than estimated, and alteplase can be safely used in patients with massive $\mathrm{PE}$ for thrombolysis. ${ }^{3,4}$ We aimed to share the results of thrombolytic treatment in patients with high-risk PE.

\section{MATERIALS AND METHODS}

We retrospectively evaluated patients admitted with PE between January 2010 and April 2018 in the Department of Pulmonary Medicine, VM Medicalpark Samsun Hospital, Samsun, Turkey. The data of patients who received thrombolytic treatment were retrospectively analyzed. The diagnosis of PE and patient selection for thrombolytic treatment were decided on using the following criteria: (1) patients aged over 17 years with symptoms suggestive of acute massive PE; (2) massive PE defined as acute PE with sustained hypotension (systolic blood pressure $<90$ $\mathrm{mmHg}$ for at least 15 minutes or requiring inotropic support, not due to a cause other than PE such as arrhythmia, hypovolemia, sepsis, or left ventricular dysfunction), pulselessness, or persistent profound bradycardia (heart rate $<40 \mathrm{bpm}$ with signs or symptoms of shock). In addition, acute right ventricular (RV) dilation, hypokinesia, and acute pulmonary arterial hypertension with paradoxical movement of the interventricular septum on echocardiography were added as criteria for thrombolytic treatment in patients with hemodynamic instability and/ or cardiopulmonary arrest; (3) PE confirmed with contrast-enhanced thorax computed tomography (CT) in appropriate patients.

\section{TREATMENT PLAN}

Alteplase (rt-PA, Actilyse ${ }^{\circledR}$; Boehringer Ingelheim, Ingelheim, Germany) was administered via the following ther- apeutic protocol: $10 \mathrm{mg}$ bolus via a central venous line and $90 \mathrm{mg}$ continuous infusion over 2 hours. All patients were continuously monitored for heart rate, systolic, diastolic, and mean systemic blood pressures, and oxygen saturation with a pulse oximeter.

The study was performed in accordance with the ethical principles in the Good Clinical Practice (GCP) guidelines and applicable local regulatory requirements, and the protocol was approved by local ethics review boards. All the patients read the patient information form about the study procedure and written informed consent was obtained.

\section{RESULTS}

A total of 72 patients were diagnosed as high-risk PE and treated with alteplase (rt-PA). The characteristics of the study population and treatment results are presented in Table 1 . The female to male ratio was $24 / 48$. The mean age of the included patients was $62.7 \pm 12.6$ (minimum 27, maximum 88) years. The diagnosis of massive PE was established with echocardiography in all patients and was confirmed via pulmonary CT angiography in 45 patients

TABLE 1. Baseline characteristics of patients with massive PE

\begin{tabular}{lc}
\hline Characteristics & Values \\
\hline Age - years, mean \pm SD (min, max) & $62.7 \pm 12.6(27,88)$ \\
Male/Female; $\mathrm{n}$ & $24 / 48$ \\
Risk factors - n (\%) & $10(13.9)$ \\
None & $15(20.8)$ \\
Surgery & $47(65.3)$ \\
Immobilization & \\
Symptoms - n (\%) & $65(90.3)$ \\
Dyspnea & $60(83.3)$ \\
Chest pain & $29(40.2)$ \\
Syncope & \\
Diagnosis - n (\%) & $27(37.5)$ \\
Echocardiography & $45(62.5)$ \\
Echocardiography with thoracic CT & $25(37.2)$ \\
Cardiopulmonary arrest & $13(18)$ \\
Drug on CPR & $4(30.7)$ \\
Alive & $19(26.2)$ \\
Overall mortality & \\
Complication - n (\%) & $6(8.3)$ \\
Minor hemorrhage & $2(2.7)$ \\
Major hemorrhage & $1(1.3)$ \\
Allergic reaction & 0 \\
Mortality due to complication & \\
\hline
\end{tabular}

CPR - Cardiopulmonary resuscitation 


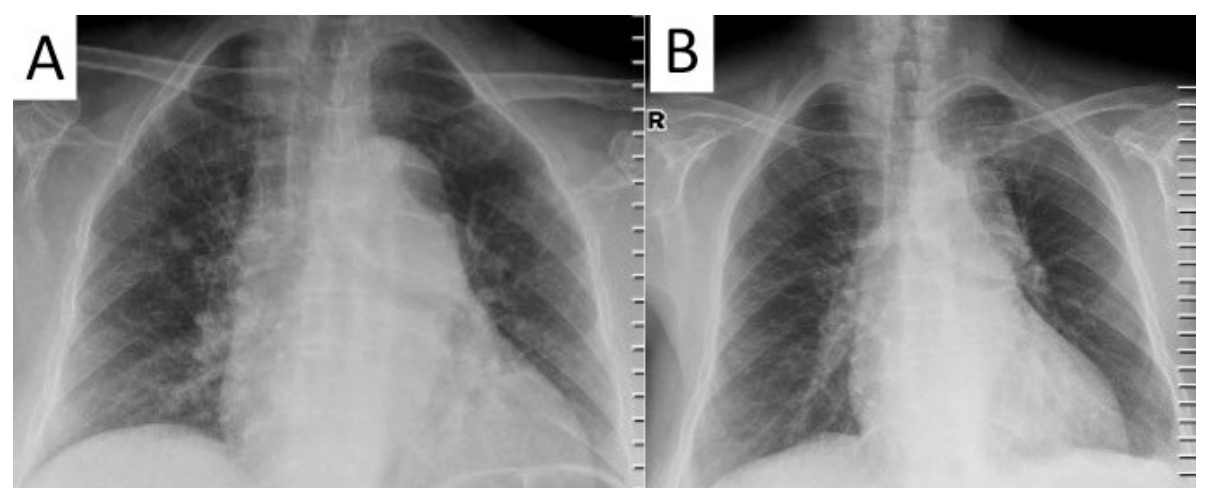

FIGURE 1. Chest radiography showing the increased cardiothoracic index at presentation (A) and 24 hours after thrombolytic treatment (B) in a patient with massive PE

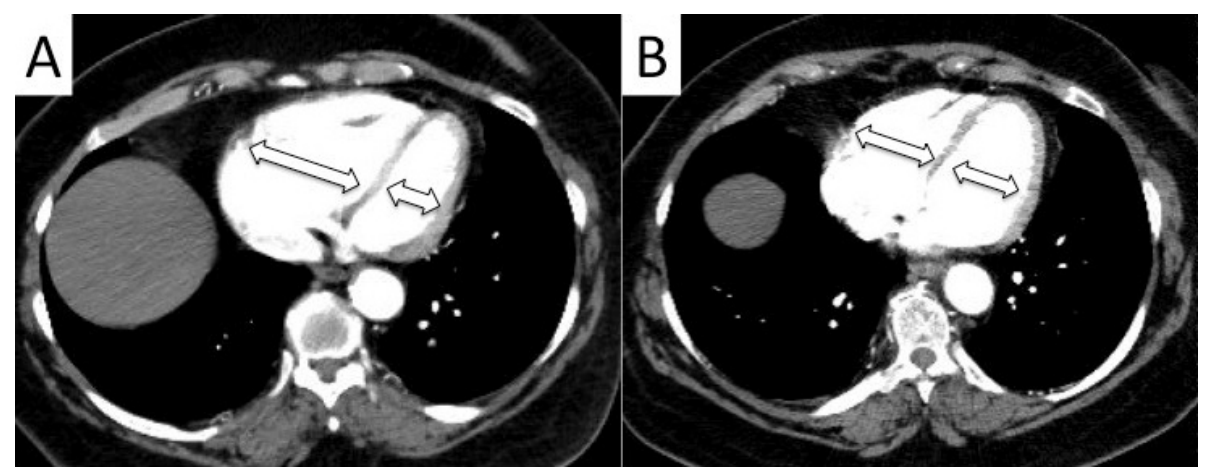

FIGURE 2. Contrast-enhanced thoracic $C T$ of a patient with massive $P E$, showing significant enlargement of the RV with a RV/LV ratio $>1$ and interventricular septal shift (A), which recovered after thrombolytic treatment (B)

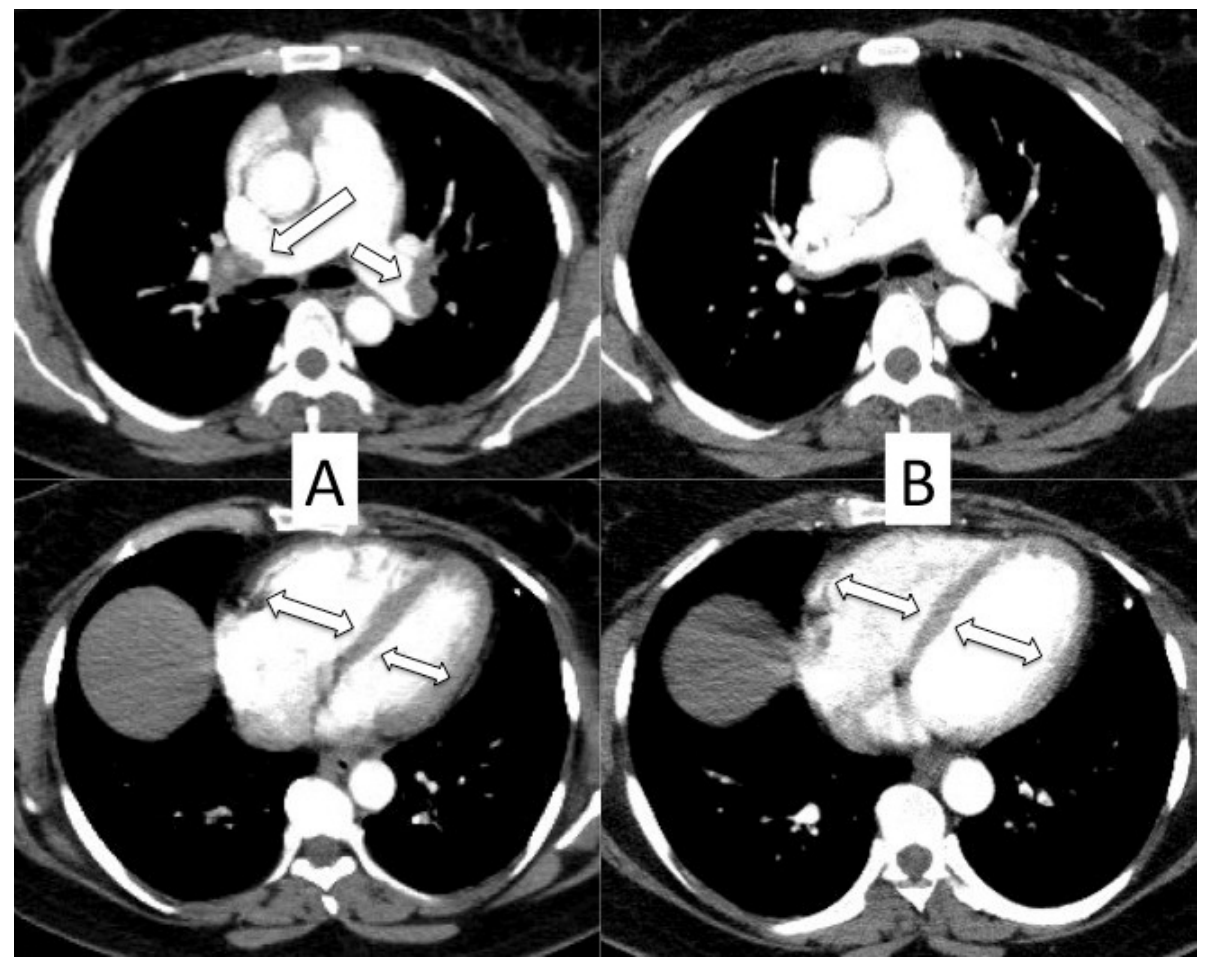

FIGURE 3. Contrast-enhanced thoracic $C T$ showing the bilateral PE in the main pulmonary arteries (A), which disappeared after the thrombolytic treatment (B) 


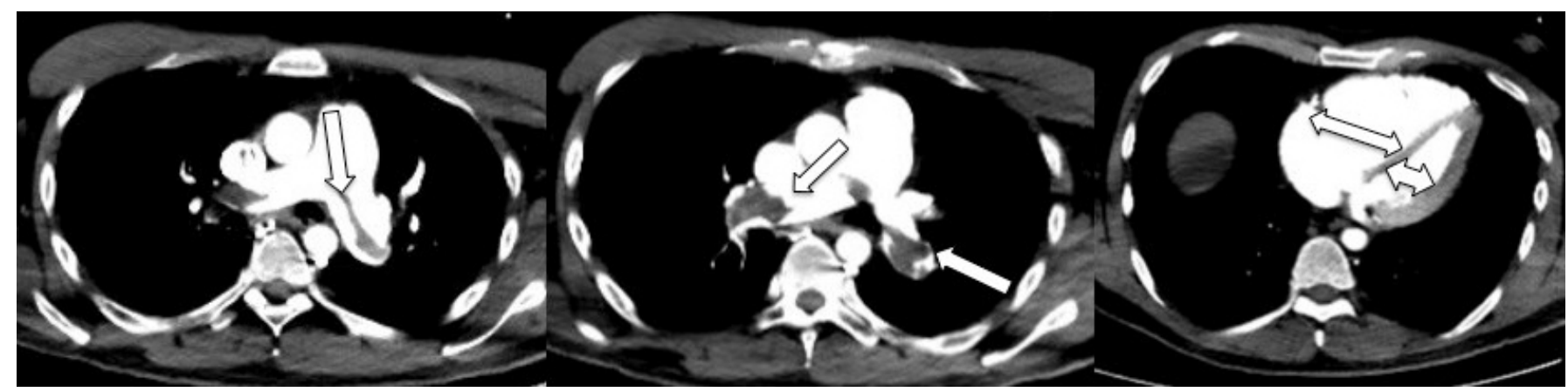

FIGURE 4. Contrast-enhanced thoracic CT showing the "saddle PE" with RV/LV >1 in a patient who died due to massive PE
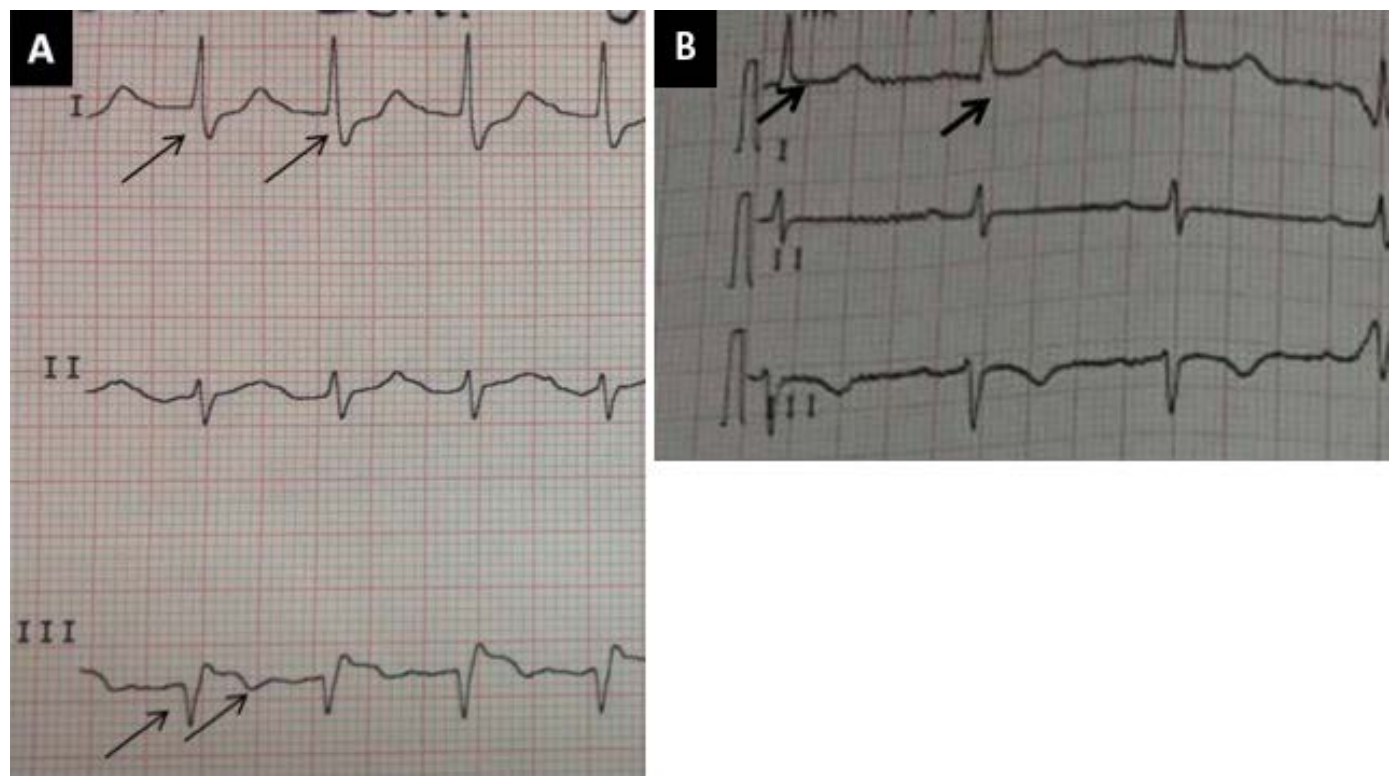

FIGURE 5. ECG tracing showing the $\mathrm{S} 1 \mathrm{Q} 3 \mathrm{~T} 3_{3}$ pattern in a patient with shock caused by massive PE (A), which rapidly recovered after thrombolytic therapy (B)

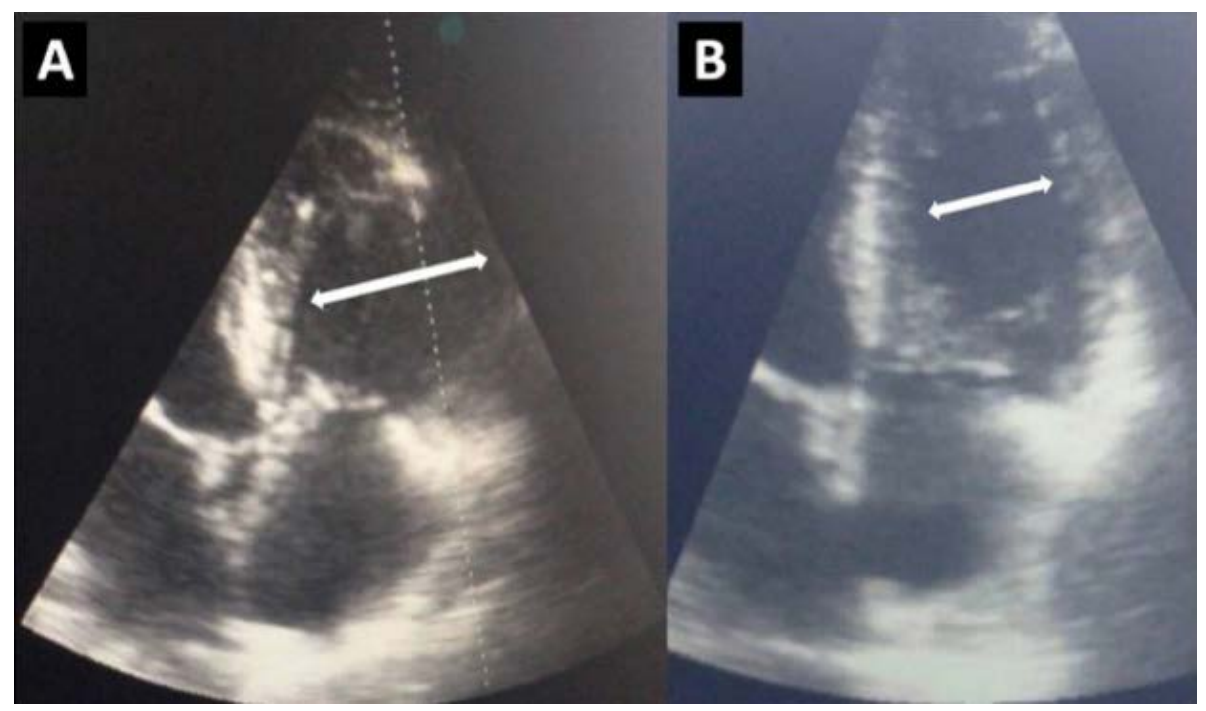

FIGURE 6. Echocardiography showing enlargement of the RV with a RV/LV ratio $>1(\mathbf{A})$ and interventricular septal shift, which normalized after thrombolytic treatment (B) in a patient with massive PE 
TABLE 2. Diagnostic approach to massive pulmonary thromboembolism in patients with shock

\begin{tabular}{|c|c|c|c|}
\hline \multirow[t]{2}{*}{ Thoracic CT } & \multicolumn{2}{|c|}{ ECG } & \multirow{2}{*}{$\begin{array}{c}\text { Echocardiography } \\
\text { RV dilatation/failure } \\
\text { Interventricular septal shift }\end{array}$} \\
\hline & $\mathrm{S}_{103} \mathrm{~T}_{3}(+)$ & $\mathrm{S}_{103 \mathrm{~T}} 3(-)$ & \\
\hline $\mathrm{RV} / \mathrm{LV}>1.0$ & A $80 \%$ & $B^{*} \quad 16 \%$ & Yes \\
\hline $\mathrm{RV} / \mathrm{LV}<1.0$ & $C^{* *} \quad 2 \%$ & $\mathrm{D}^{* * *} \quad 2 \%$ & N/A \\
\hline
\end{tabular}

$\mathrm{B}^{*}$ and $\mathrm{D}^{* * *}$ - patients with cardiopulmonary arrest; $\mathrm{C}^{* *}-\mathrm{CT}$ and clinical (hypotension) diagnosis of massive PTE; N/A - not available

$(62.5 \%)$ who presented an appropriate clinical status for this imaging technique (Figures 1-6). Most common symptoms were dyspnea (90.3\%), chest pain (83.3\%), and syncope $(40.2 \%)$. The $\mathrm{S}_{103 \mathrm{~T} 3}$ pattern was noted in $82 \%$ of patients on the 12-lead electrocardiography, which rapidly recovered after thrombolytic therapy (Figure 5). The diagnostic approach used in our institution is shown in Table 2. Cardiopulmonary arrest was observed in 37.2\% (n = 25) of patients, and thrombolytic treatment was given during cardiopulmonary resuscitation in $18 \%(n=13)$ of them. The survival rate of patients with cardiac arrest who received thrombolysis in the emergency department was $30.7 \%(n=4)$.

The complications of rt-PA treatment included minor hemorrhages in $6.4 \%(n=5)$, major hemorrhages in $2.7 \%$ $(\mathrm{n}=2)$, and allergic reactions in $1.3 \%(\mathrm{n}=1)$ of patients. None of the patients had deceased as a complication of thrombolytic treatment. The overall mortality rate was $26.2 \%(n=19)$, and $12.5 \%(n=9)$ of the patients died in first 24 hours after thrombolysis.

\section{DISCUSSIONS}

The effect of thrombolytic therapy on mortality and the frequency of recurrent thromboembolism remain questionable. Persistent hypotension or shock (defined as systolic blood pressure $<90 \mathrm{mmHg}$ or a decrease in the systolic blood pressure by $\geq 40 \mathrm{mmHg}$ from baseline) due to acute PE is the only widely accepted indication for systemic thrombolysis. ${ }^{5}$ Evidence from randomized and retrospective observational studies in patients with acute PE indicates that thrombolytic therapy leads to early hemodynamic improvement, but at a cost of increased major bleeding. Streptokinase, urokinase (also known as urinary plasminogen activator), and alteplase are the only thrombolytic agents with this indication. A case-control study assessed 62 adults for risk factors that might be associated with bleeding after treatment with alteplase. The investigators found that patients with major bleeding more often had recent major surgery, an INR greater than $1.7(\mathrm{p}=0.008)$, and one or more risk factors for bleeding compared with those without major bleeding. Other clinical data have shown that patients with a lower threshold for bleeding during thrombolytic therapy are more likely to have a history of recent major surgery, trauma, pregnancy, cardiopulmonary resuscitation, or an invasive procedure. These findings underscore the importance of considering a patient's bleeding potential before administering thrombolytics. ${ }^{6}$ In our study, cardiopulmonary arrest was seen in $37.2 \%$ of patients, while thrombolytic treatment was administered in $18 \%$ of patients, with a survival rate of $30.7 \%(n=4)$. Our study also assessed the rate of complications due to thrombolysis, including major (2.7\%) and minor bleeding $(6.4 \%)$ as well as allergic reactions (1.3\%), while none of the study subjects had deceased due to administration of thrombolytic treatment. The overall mortality rate was $26.2 \%$ in the study population, and $12.5 \%$ within the first $24 \mathrm{~h}$ after thrombolysis.

In a previous publication, we have reported a case series which included patients with massive PE who had been administered thrombolysis during cardiopulmonary resuscitation. ${ }^{3}$ In the case series, the mortality rate was $50 \%$, with higher rates of minor bleeding (14\%) but with no major hemorrhagic complications. Furthermore, the thrombolytic treatment was not associated with any fatal hemorrhage complication. ${ }^{3}$

Despite its low sensitivity and specificity, ECG tracing adds diagnostic value in determining the extent of the thrombotic burden and supporting the finding of right ventricular dysfunction as seen by echocardiography. Sinus tachycardia, supraventricular tachyarrhythmias, S103T3 pattern, new complete or incomplete right bundle branch block, and P pulmonale are some of the ECG patterns described in association with PE. In the PIOPED database, $30 \%$ of patients were noted to have a normal ECG, but the most common ECG finding was noted to be non-specific abnormalities of the ST segment or T wave in $49 \%$ of their population. Inverted $\mathrm{T}$ waves in leads $\mathrm{V} 1-\mathrm{V} 4$ may be present, especially in the setting of massive PE. In one study, this pattern was shown to correlate with the severity of PE. ${ }^{7,8}$

The most common symptoms were dyspnea (90.3\%), chest pain $(83.3 \%)$, and syncope $(40.2 \%)$. The $\mathrm{S}_{103 \mathrm{~T}} 3$ pattern was noted in $82 \%$ of patients on the ECG tracings. Also, we noted that the $\mathrm{S}_{103 \mathrm{~T}} 3$ pattern had rapidly improved after thrombolytic therapy in 24 hours. Dogan et al. analyzed the role of $\mathrm{CT}$ in the diagnosis of acute and chronic PE. ${ }^{9}$ International multicenter echocardiographic 
studies have shown that in PE patients, right ventricular dysfunction is a major determinant of short-term mortality. RV dysfunction can also be recognized on CT, by measuring the ratio of RV/LV diameters or by volumetric measurements that can be obtained with or without ECG-gating. Several multidetector CT studies have shown the prognostic value of RV dysfunction that may help to identify patients at risk and may facilitate selecting the therapeutic strategies. ${ }^{10-16} \mathrm{~A} \mathrm{RV} / \mathrm{LV}$ diameter ratio cutoff value of $>1.0$ is commonly considered to represent RV dysfunction and has been shown to predict short-term adverse outcome and mortality rates. Axial and fourchamber view measurements of RV/LV diameter ratio $>1.0$ are comparable for predicting 30 -day mortality. ${ }^{14-18}$ These results were consistent with our study. We found that the diagnostic accuracy of thoracic CT in identifying RV dysfunction on the basis of a RV/LV diameter ratio $>1.0$ was $95 \%$ as related to echocardiography.

\section{CONCLUSIONS}

We concluded that risk factors, ECG, and echocardiography are key indicators for the suspicion of massive PE in patients with hemodynamic shock. If there is any sign of acute PE, echocardiography should be used during cardiopulmonary arrest/instability, and alteplase should be given to patients with suspected massive PE. In patients with massive PE, mortality rates associated with thrombolytic treatment are not higher than those associated with anticoagulant therapy in the absence of thrombolytic treatment. Based on our experience, early thrombolytic therapy is a life-saving intervention in patients with diagnosed and/or suspected massive PE.

\section{CONFLICT OF INTEREST}

The authors have no direct conflicts of interest to declare. This research work was supported by VM Medicalpark Samsun Hospital, Samsun, Turkey.

\section{DATA AVAILABILITY}

The research article data used to support the findings of this study are available from the corresponding author upon request.

\section{REFERENCES}

1. Jaff MR, McMurtry MS, Archer SL, et al. Management of massive and submassive pulmonary embolism, iliofemoral deep vein thrombosis, and chronic thromboembolic pulmonary hypertension: a scientific statement from the American Heart Association. Circulation. 2011;123:1788-1830. doi: 10.1161/CIR.ob013e318214914f.

2. Marti C, John G, Konstantinides S, et al. Systemic thrombolytic therapy for acute pulmonary embolism: a systematic review and meta-analysis. Eur Heart J. 2015;36:605-614. doi: 10.1093/ eurheartj/ehu218.

3. Dirican A, Ozkaya S, Atas AE, et al. Thrombolytic treatment (alteplase; rt-Pa) in acute massive pulmonary embolism and cardiopulmonary arrest. Drug Des Devel Ther. 2014;8:759763. doi: 10.2147/DDDT.S61679.

4. Wan S, Quinlan DJ, Agnelli G, Eikelboom JW. Thrombolysis compared with heparin for the initial treatment of pulmonary embolism. Circulation. 2004;110:744-749. doi: 10.1161/01. CIR.0000137826.09715.9C.

5. Kearon C, Akl EA, Comerota AJ, et al. Antithrombotic therapy for VTE disease: Antithrombotic Therapy and Prevention of Thrombosis, 9th ed: American College of Chest Physicians Evidence-Based Clinical Practice Guidelines. Chest. 2012;141:e419S. doi: 10.1378/chest.11-2301.

6. Martin C, Sobolewski K, Bridgeman P, et al. Systemic Thrombolysis for Pulmonary Embolism: A Review. P T. 2016;41:770-775.

7. Sekhri V, Mehta N, Rawat N, Lehrman SG, Aronow WS. Management of massive and nonmassive pulmonary embolism. Arch Med Sci. 2012;8:957-969.

8. Stein PD, Terrin ML, Hales CA, et al. Clinical, laboratory, roentgenographic, and electrocardiographic findings in patients with acute pulmonary embolism and no pre-existing cardiac or pulmonary disease. Chest. 1991;3:598-603. doi: 10.1378/chest.100.3.598.

9. Dogan H, Roos AD, Geleijins J, Huisman MV, Kroft LJ. The role of computed tomography in the diagnosis of acute and chronic pulmonary embolism. Diagn Interv Radiol. 2015;21:307-316. doi: 10.5152/dir.2015.14403.

10. Quiroz R, Kucher N, Schoepf UJ, et al. Right ventricular enlargement on chest computed tomography: prognostic role in acute pulmonary embolism. Circulation. 2004;109:24012404. doi: 10.1161/01.CIR.0000129302.90476.BC.

11. Reid JH, Murchison JT. Acute right ventricular dilatation: a new helical CT sign of massive pulmonary embolism. Clin Radiol. 1998;53:694-698. doi: 10.1016/s0009-9260(98)80297-3.

12. Ribeiro A, Lindmarker $P$, Juhlin-Dannfelt A, Johnsson $H$, Jorfeldt L. Echocardiography Doppler in pulmonary embolism: right ventricular dysfunction as a predictor of mortality rate. Am Heart J. 1997;134:479-487.

13. Dogan H, Kroft LJ, Huisman MV, van der Geest RJ, de Roos A. Right ventricular function in patients with acute pulmonary embolism: analysis with electrocardiography-synchronized multi-detector row CT. Radiology. 2007;242:78-84. doi: 10.1148/radiol.2421052089.

14. Lu MT, Cai T, Ersoy H, et al. Comparison of ECG-gated versus non-gated CT ventricular measurements in thirty patients with acute pulmonary embolism. Int J Cardiovasc Imaging. 2009;25:101-107. doi: 10.1007/s10554-008-9342-0.

15. Van der Bijl N, Klok FA, Huisman MV, et al. Measurement of right and left ventricular function by ECG-synchronized CT scanning in patients with acute pulmonary embolism: usefulness for predicting short-term outcome. Chest. 2011;140:1008-1015. doi: 10.1378/chest.10-3174. 
16. Kang DK, Thilo C, Schoepf UJ, et al. CT signs of right ventricular dysfunction: prognostic role in acute pulmonary embolism. JACC Cardiovasc Imaging. 2011;4:841-849. doi: 10.1016/j.jcmg.2011.04.013.

17. Kumamaru KK, Lu MT, Ghaderi NS, Hunsaker AR. Right ventricular enlargement in acute pulmonary embolism derived from CT pulmonary angiography. Int J Cardiovasc Imaging. 2013;29:705-708. doi: 10.1007/s10554-012-0126-1.

18. Becattini C, Agnelli G, Vedovati MC, et al. Multidetector computed tomography for acute pulmonary embolism: diagnosis and risk stratification in a single test. Eur Heart J. 2011;32:1657-1663. doi: 10.1093/eurheartj/ehr108. 